\title{
Bacteriologic Profile and Antimicrobial Resistance of Blood Culture Isolates of Septicemic Patients from Various Intensive Care Units in a Teaching Tertiary Care Institute of Haryana, India
}

\author{
Jyoti Sangwan ${ }^{1 *}$, Pratibha Mane ${ }^{1}$, Prakriti Vohra ${ }^{1}$, Sumit Lathwal $^{2}$ and A.K. Malik ${ }^{1}$ \\ ${ }^{1}$ Department of Microbiology, SHKM GMC, Mewat, Haryana, India \\ ${ }^{2} \mathrm{ADH}, 12$ CORPS,C/O 56 APO, India \\ *Corresponding author
}

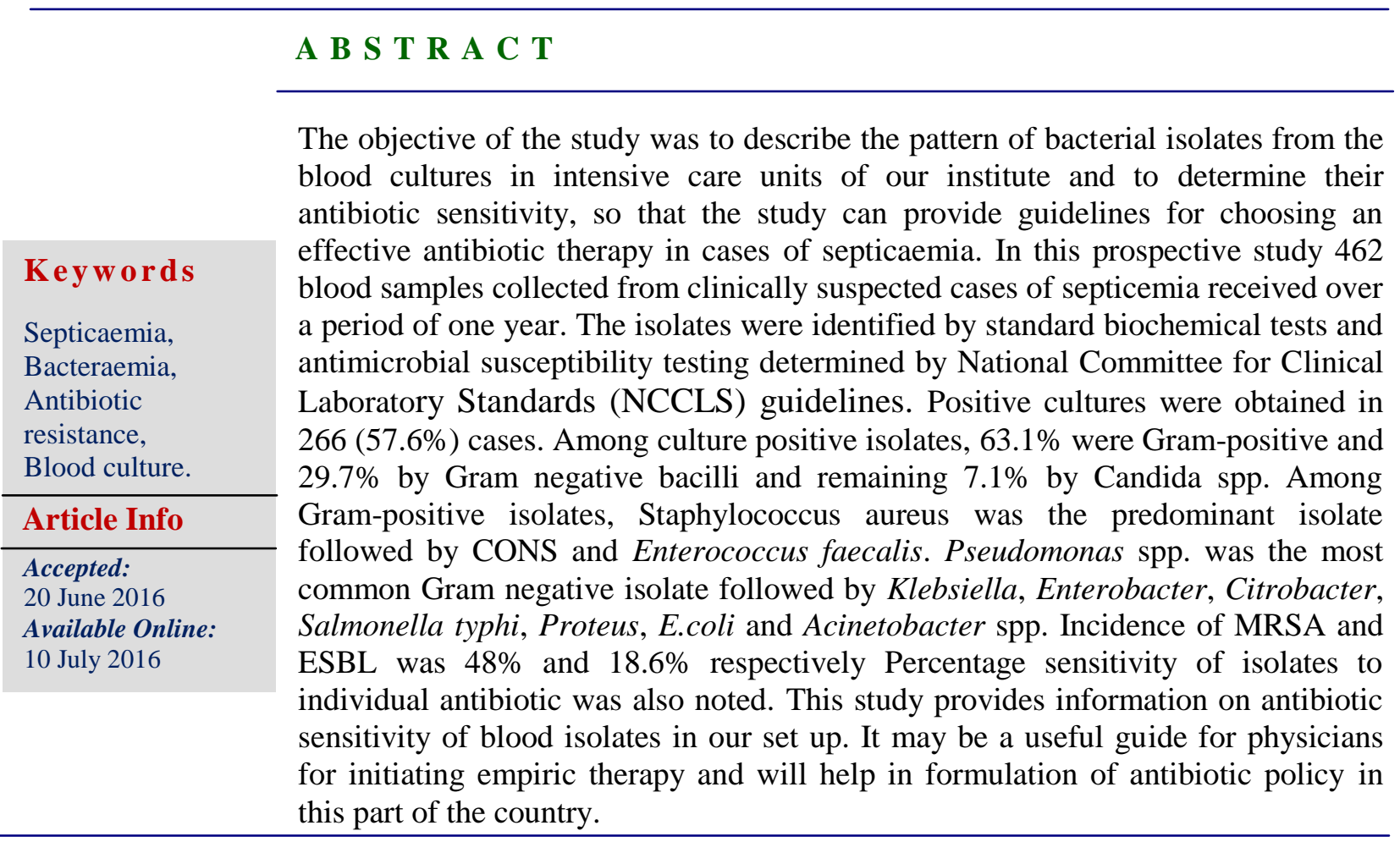

\section{Introduction}

Infection rates in intensive care units (ICUs) have been found to be the highest among all hospital acquired infections in various multicentre studies carried out in the United States of America (USA) and Europe (Vincent JL et al., 1995) and (Jarvis WR et al., 1991). This occurs due to the use of large numbers of invasive monitoring devices, endotracheal and tracheostomy tubes along with the patient factors including extremes of age, immunocompromise state, malnutrition and severe underlying disease. Also a high incidence of cross infection is seen in ICUs (Weber DJ et al., 1999). These ICU infections are costly to treat, lead to prolong ICU stay and increased mortality rates (Eggimann, et al., 2001). 
Sepsis is a common and threatening infection in the intensive care unit, where up to $35 \%$ of patients develop such a condition at some point during their stay. The associated mortality is $27 \%$ but exceeds $50 \%$ in cases of septic shock (Vincent, et al., 2006).

Blood cultures represent an important diagnostic tool, although they detect bacteremia in only about $50 \%$ of patients clinically suspected of having sepsis (BrunBuisson, et al., 1995), with an even lower rate of positivity when drawn in the presence of ongoing antibiotic therapy (Bergmans, et al., 1997). The presence of a blood pathogen represents a negative prognostic factor, but the isolation of such pathogen is important for verifying the appropriateness of antibiotic therapy, which in turn is known to reduce morbidity and mortality (Grace, et al., 2001). Also, falsepositive results due to bacterial contaminants may lead to unnecessary antibiotic therapy, longer hospital stays and selection of resistant microorganisms (Bates, et al., 1991).

There is wide diversity between institutions in the prevalence of pathogens and in their antimicrobial susceptibility. There is also variation in the frequency and types of infections among different subsets of patients within the same ICU. To produce effective empirical antibiotic protocols for individual ICUs, knowledge of common organisms and their sensitivity patterns is essential. The aim of this study was to determine the rate of positive cultures and to identify the common organisms and their susceptibility patterns from bacteremic patients in the Intensive Care Units, of our institute over a one-year period. Knowing the most frequent pathogenic microorganisms that colonise or infect such type of patient allow for effective empirical antibiotic treatment taking into account microorganisms' susceptibility and resistance patterns. A general increase in bacterial resistance rates has been reported around the world, giving rise to multi resistant strains within hospitals and thereby generating serious problems for health-care institutions.

\section{Materials and Methods}

This study was carried out from January 2014 to December 2014 in a tertiary care institute. Consecutive blood samples received from various ICUs were included, clinical details of patients were noted. Processing of samples was done immediately after receiving in department of microbiology. $5 \mathrm{ml}$ of blood was collected from each adult patient using strict aseptic precautions, and inoculated immediately into $50 \mathrm{ml}$ of 'Brain Heart Infusion' (BHI) broth with $0.025 \%$ of sodium polyanthol sulphonate as anticoagulant (HI media). In paediatric cases $1-2 \mathrm{ml}$ of blood was inoculated in $5-10 \mathrm{ml}$ of BHI broth. The broths were subcultured on blood agar and MacConkey agar after overnight incubation. A negative result was followed-up by examining the broth daily and doing a final subculture at the end of seventh day. Positive growth was identified by Gram staining, colony characteristics, and standard biochemical tests.

The antibiotic sensitivity testing of all isolates was performed by Kirby Bauer's disc diffusion method on Muller Hinton agar and interpreted as per CLSI guidelines and classified as sensitive and resistant (NCCLS. 2002.). Standard antibiotics such as ampicillin $(30 \mu \mathrm{g})$, amoxyclav $(30 \mu \mathrm{g})$, ceftriaxone $(30 \mu \mathrm{g})$, ceftazidime $(30 \mu \mathrm{g})$, gentamicin $(10 \mu \mathrm{g}$ and $120 \mu \mathrm{g})$, imipenem $(10 \mu \mathrm{g})$, cefoxitin $(30 \mu \mathrm{g})$, linezolid $(30 \mu \mathrm{g})$, vancomycin $(30 \mu \mathrm{g})$, piperacillin $(100 \mu \mathrm{g})$, 
chloramphenicol $\quad(30 \quad \mu \mathrm{g})$,Ciprofloxacin $(5 \mu \mathrm{g})$, Erythromycin $(10 \mu \mathrm{g})$, Netilmicin $(30$ $\mu \mathrm{g})$, Colistin(10 $\mu \mathrm{g})$, Teicoplanin(30 $\mu \mathrm{g})$, Amikacin $\quad(30 \mu \mathrm{g}), \quad$ Piperacillin Tazobactam(100/10 $\mu \mathrm{g})$, Tigecyclin(30 $\mu \mathrm{g})$, Polymyxin $\mathrm{B}(10 \mu \mathrm{g})$ Clindamycin $(2 \mu \mathrm{g})$, Cotrimoxazole (Trimethoprim $1.25 \mu \mathrm{g} /$ Sulfomethoxazole $23.75 \mu \mathrm{g})$ were tested. Escherichia coli ATCC 25922, Pseudomonas aeruginosa ATCC 27853, Staphylococcus aureus ATCC 25923 and E. faecalis ATCC 29212 were used for quality control. All the culture media, biochemical media and antibiotics used were obtained from Hi Media.

Detection of Methicillin resistant Staphylococcus aureus (MRSA) was done with Cefoxitin $(30 \mu \mathrm{g})$ disc. Extended spectrum betalactamase (ESBL) production was detected with the help of Ceftazidime $(30 \mu \mathrm{g})$ and Ceftazidime + Clavulanic acid $(30 / 10 \mu \mathrm{g})$. Results obtained were analyzed by counts and percentages using MS Excel, 2007 version.

\section{Results and Discussion}

A total of 462 samples were received during the study period out of which $266(57.6 \%)$ were culture positive. Out of these 266 positive samples $226(84.9 \%)$ had $\mathrm{C}$ reactive protein (CRP) value more than $6.4 \mathrm{mg} / \mathrm{dl}$. Most infections were due to a single organism, while blood of two neonates grew Klebsiella pneumoniae and Pseudomanas aeruginosa both. The breakup of 462 samples received from various ICUs is shown in Figure 1, among which Paediatric ICU with 246 samples (53.2\%) was the major contributor followed by Neonatal ICU with 190 samples $(41.1 \%)$, Medical ICU with 21 samples $(4.5 \%)$ and Surgical ICU with 5 samples $(1.2 \%)$.The various bacterial isolates obtained were compared among paediatric patients versus adults which is depicted in Figure 2 and Table 2.
A total of 266 organisms were isolated. The commonest organism was Staphylococcus aureus $(33.5 \%)$ followed by Coagulase Negative Staphylococcus spp i.e CONS (25.9\%), Enterobacteriaceae (18.4\%), Pseudomonas (9.4\%), non albicans Candida (6.8\%), Enterococcus (3.4\%), Acinetobacter $(1.9 \%)$ and Candida albicans and Meningococcus both $(0.04 \%)$ as shown in Figure 3.

In Neonatal ICU from total 190 samples the positivity rate was $66.3 \%$ (126/190). Similarly, in Paediatric ICU from total 246 samples the positivity rate was $50.4 \%$ (124/246). The sample positivity rate from MICU and SICU was 58.3\% (14/21) and $40.0 \% \quad(2 / 5)$ respectively. The most predominant bacteria isolated from all the ICUs was Staphylococcus aureus followed by CONS, Enterobacteriaceae and Pseudomonas. Non albicans Candida and Candida albicans were mainly isolated only from patients of NICU and PICU. The incidence and distribution of isolates from various ICUs is shown in Table 1.

The most predominant gram positive bacterium isolated was Staphylococcus aureus from all ICUs followed by CONS and Enterococci. Though Enterobacteriaceae was the most predominant family isolated among gram negative bacteria (GNB), Pseudomonas was the most predominant single Gram negative organism from all ICUs except NICU where Klebsiella pneumoniae was the most common GNB isolated. Enterobacteriaceae isolated were Klebsiella pneumoniae, E.coli, Serratia marcescens, Proteus mirabilis, Citrobacter spp, Enterobacter spp and Salmonella typhi.

The distribution of pathogens in PICUs differed from that we have observed in adults, and within the PICUs and changed with age. Isolation of CONS was 
significantly higher in children when compared to adults $(\mathrm{p} \leq 0.05)$. Similarly, $E$. fecalis was isolated only from children. Incidence of $S$. aureus was similar in adults and children. Similarly, Gram-negative bacteria isolated were also comparable. Fungal pathogens were reported more commonly in bloodstream infections in children, but much less frequently in adult ICUs (7\% vs 0) (Table II).

The antibiogram of gram positive cocci revealed that resistance to Vancomycin, Linezolid and Teicoplanin is yet to be seen in our set up. $S$. aureus shows very high resistance to Amoxycillin, Amoxy Clav, Cotrimoxazole and Ciprofloxacin (sensitivity ranging from 10-30\%).S. aureus was fairly sensitive to Amino glycoside group (resistance ranging from 25-35\%). Also S.aureus was sensitive to Macrolide group (resistance 30-40\%). Enterococcus was highly resistant to Penicillins, Cotrimoxazole and Macrolides(resistance ranging from $70-80 \%$ ). Senitivity to Gentamicin was good in Enterococcus. (Table III)

Gram negative bacteria of Enterobacteriaceae except Salmonella typhi were most susceptible to Imipenem (sensitivity 90-100\%), only two isolates of Klebsiella spp showed resistance. Enterobacteriaceae showed very high resistance to Ampicillin, Amoxy Clav and Ciprofloxacin (sensitivity ranging from 5$30 \%$ ). Resistance to Cephalosporins ranged from $30-50 \%$ in these isolates. Sensitivity to polymixin B and Colistin was $100 \%$ as all Enterobacteriaceae was sensitive (Table IV).

In Salmonella typhi Ceftriaxone and ciprofloxacin were very effective with no resistance as none of the 4 isolates showed resistance. Also no resistance was seen against Chloramphenicol in our institute. All the four isolates were reisistant to Ampicillin (100\% resistance) and 3/4 isolates were resistant to Cotrimoxazole (75\% resistance) as shown in (Table IV).

No resistance against Imipenem, Polymixin B and Colistin was reported in Pseudomonas and Acinetobacter spp isolated from blood (Table IV). Piperacillin and Netilmicin are no more effective against Pseudomonas as resistance is high being 60-75\%, Piperacillin Tazobactam combination being slightly better (resistance 56\%). Resistance to Ceftazidime is also on the rise in our set up being $72 \%$. Sensitivity to Amikacin and Gentamicin is still there in Pseudomonas being 60 and $64 \%$ respectively.

Out of 19 Candida spps, 18 were non albicans candida and one was Candida albicans. The antifungal susceptibility testing was not done. One Meningococcus isolated was sensitive to all antibiotics tested.

Incidence of MRSA and ESBL was $48 \%$ and $18.6 \%$ respectively.

Sepsis, a systemic inflammatory response to infection, is an important cause of morbidity and mortality in critically ill patients, resulting in frequent diagnostic testing, greater prescription of antibiotics, and increased length of hospitalization. Bacteremia (including fungemia) in critically ill patients has an average attributable mortality of 26\%, (Pittet, et al., 1994). However, the microbiologic diagnosis of bacteremia acquired after admission is often difficult in the ICU, complicated by the fact that many critically ill patients receive empiric antibiotic therapy. In fact, antibiotic consumption is 10 times greater in ICUs than on general hospital wards (Bergmans, et al., 1997). Pediatric intensive care units (PICUs) differ 
from adult intensive care units (ICUs) in a number of ways, apart from the age of their patients. First, they are usually multidisciplinary, because there are too few patients to justify separate medical and surgical units. Second, they frequently lack the physical barriers between patients now commonly present in adult ICUs. Third, fewer children than adults in ICUs have chronic or degenerative organ system disorders and probably the majority of children in PICUs will, if successfully treated, return to a normal productive life.

Our analysis suggests that the epidemiology of nosocomial infections in PICUs and NICU differs from that seen in adult critical care units. The distribution of infection sites lies between what we have previously reported in neonatal ICUs and adult medical ICUs (Gaynes et al., 1996). First, primary bloodstream infections were the most common sites of infection, followed by pneumonia and UTIs. In adult medical ICUs, UTIs were most frequently reported. In neonatal ICUs, bloodstream infections were an even greater proportion of all infections than in pediatric units. We have also observed high rate of positive blood culture in children compared to adults (50$66 \%$ in children vs $40-58 \%$ in adults) though this difference was not statistically significant $(\mathrm{p} \geq 0.05)$.

The distribution of pathogens in PICUs differed from that we have observed in adults, and within the PICUs and changed with age. These differences in pathogen distribution seem likely to be, in part, an effect of age but also to reflect other patientrelated factors, including underlying medical and surgical conditions, previous hospital stay, and antibiotic exposure.

Table.1 Incidence and distribution of microorganisms isolated from blood cultures from various ICUs.

\begin{tabular}{|l|l|l|l|l|l|}
\hline \multicolumn{1}{|c|}{ Isolate } & $\begin{array}{l}\mathrm{NICU} \\
\mathrm{n}=126\end{array}$ & $\begin{array}{l}\mathrm{PICU} \\
\mathrm{n}=124\end{array}$ & $\begin{array}{l}\text { MICU } \\
\mathrm{n}=14\end{array}$ & $\begin{array}{l}\text { SICU } \\
\mathrm{n}=2\end{array}$ & $\begin{array}{l}\text { TOTAL } \\
\mathrm{n}=266\end{array}$ \\
\hline CONS & $30(23.8)$ & $37(29.8)$ & $2(14.3)$ & 0 & $69(25.9)$ \\
\hline S.aureus & $33(26.2)$ & $50(40.3)$ & $5(35.7)$ & 1 & $89(33.4)$ \\
\hline Enterococcus & $6(4.8)$ & $3(2.4)$ & 0 & 0 & $9(3.4)$ \\
\hline Pseudomonas & $10(7.9)$ & $12(9.7)$ & $3(21.4)$ & 0 & $25(9.4)$ \\
\hline Acinetobacter & $2(1.5)$ & $3(2.4)$ & 0 & 0 & $5(2.0)$ \\
\hline Salmonella spp. & 0 & $2(1.6)$ & $2(14.3)$ & 0 & $4(1.5)$ \\
\hline E. coli & $1(0.7)$ & $2(1.6)$ & 0 & $1(50)$ & $4(1.5)$ \\
\hline Klebsiella spp. & $15(11.9)$ & $3(2.4)$ & 0 & 0 & $18(6.8)$ \\
\hline Citrobacter & $2(1.5)$ & $6(4.8)$ & $2(14.3)$ & 0 & $10(3.8)$ \\
\hline Serratia marcescens & $4(3.2)$ & 0 & 0 & 0 & $4(1.5)$ \\
\hline Enterobacter spp & $6(4.8)$ & $3(2.4)$ & 0 & 0 & $9(3.4)$ \\
\hline C. albicans & $1(0.8)$ & 0 & 0 & 0 & $1(0.4)$ \\
\hline Non albicans Candida & $16(12.7)$ & $2(1.6)$ & 0 & 0 & $18(6.8)$ \\
\hline Meningococcus & 0 & $1(0.8)$ & 0 & 0 & $1(0.4)$ \\
\hline & & & & &
\end{tabular}


Table.2 Comparison of isolates $\mathrm{b} / \mathrm{w}$ children and adults

\begin{tabular}{|l|l|l|}
\hline & $\begin{array}{l}\text { NEONATES/CHILDREN } \\
250\end{array}$ & $\begin{array}{l}\text { ADULTS } \\
16\end{array}$ \\
\hline CONS & $67(26.8)$ & $2(12.5)$ \\
\hline S.aureus & $83(33.2)$ & $6(37.5)$ \\
\hline Enterococcus & $9(3.6)$ & 0 \\
\hline Pseudomonas & $22(8.8)$ & $3(18.7)$ \\
\hline Acinetobacter & $5(2.0)$ & 0 \\
\hline Enterobacteriaceae & $44(17.6)$ & $5(31.2)$ \\
\hline C. albicans & $1(0.4)$ & 0 \\
\hline $\begin{array}{l}\text { Non albicans } \\
\text { Candida }\end{array}$ & $18(7.2)$ & 0 \\
\hline Meningococcus & $1(0.4)$ & 0 \\
\hline
\end{tabular}

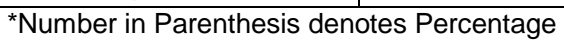

Table.3 Resistance pattern of Gram-positive isolates.

\begin{tabular}{|c|c|c|}
\hline Antibiotic & $\begin{array}{c}\text { Staphylococcus spp } \\
\mathrm{N}=89\end{array}$ & $\begin{array}{c}\text { Enterococcus spp } \\
\mathrm{N}=9\end{array}$ \\
\hline Penicillin & $78(87.6)$ & $7(77.8)$ \\
\hline Amoxycillin & $79(88.7)$ & $0(100)$ \\
\hline Amoxy Clav & $70(78.6)$ & $0(100)$ \\
\hline Cefoxitin & $52(58.4)$ & - \\
\hline Erythromycin & $39(43.8)$ & $4(44.4)$ \\
\hline Gentamicin & $32(35.9)$ & - \\
\hline Amikacin & $25(28.1)$ & - \\
\hline Ciprofloxacin & $46(51.6)$ & - \\
\hline Clindamycin & $36(40.4)$ & $3(33.3)$ \\
\hline Gentamicin 120 & $21(23.6)$ & $2(22.2)$ \\
\hline Vancomycin & $0(0)$ & $0(0)$ \\
\hline Linezolid & $0(0)$ & $0(0)$ \\
\hline Teicoplanin & $0(0)$ & $0(0)$ \\
\hline
\end{tabular}

*Number in Parenthesis denotes Percentage 
Table.4 Resistance pattern of Gram-negative isolates.

\begin{tabular}{|c|c|c|c|c|}
\hline Antibiotic & $\begin{array}{c}\text { Enterobacteriaceae } \\
\text { except Salmonella typhi } \\
\mathrm{N}=45\end{array}$ & $\begin{array}{c}\text { Salmonella } \\
\text { typhi } N=4\end{array}$ & $\begin{array}{c}\text { Pseudomonas } \\
\mathrm{N}=25\end{array}$ & $\begin{array}{c}\text { Acinetobacter } \\
\mathrm{N}=5\end{array}$ \\
\hline Ampicillin & $43(95.6)$ & $4(100)$ & - & $5(100)$ \\
\hline Ceftriaxone & $24(53.3)$ & $0(0)$ & - & $3(60)$ \\
\hline Ceftazidime & $27(60)$ & - & $18(72)$ & $2(40)$ \\
\hline Gentamicin & $14(31.1)$ & - & $9(36)$ & $1(20)$ \\
\hline Amikacin & $10(22.2)$ & - & $10(40)$ & $1(20)$ \\
\hline Ciprofloxacin & $37(82.2)$ & $0(0)$ & $17(68)$ & $4(80)$ \\
\hline Cotrimoxazole & $39(86.7)$ & $3(75)$ & - & - \\
\hline Chloramphenicol & - & $0(0)$ & - & - \\
\hline Piperacillin & - & - & $19(76)$ & - \\
\hline Netilmicin & - & - & $16(64)$ & - \\
\hline Piperacillin + & & - & $14(56)$ & $0(0)$ \\
\hline tazobactam & - & - & $0(0)$ & - \\
\hline Imipenem & $0(0)$ & - & $0(0)$ & $2(40)$ \\
\hline Polymyxin B & $0(0)$ & - & $0(0)$ & $0(0)$ \\
\hline Tigecyclin & $0(0)$ & & $0(0)$ & \\
\hline Colistin & $0(0)$ & & \\
\hline
\end{tabular}

${ }^{*}$ Number in Parenthesis denotes Percentage

Fig.1 Sample Distribution from various ICUs.

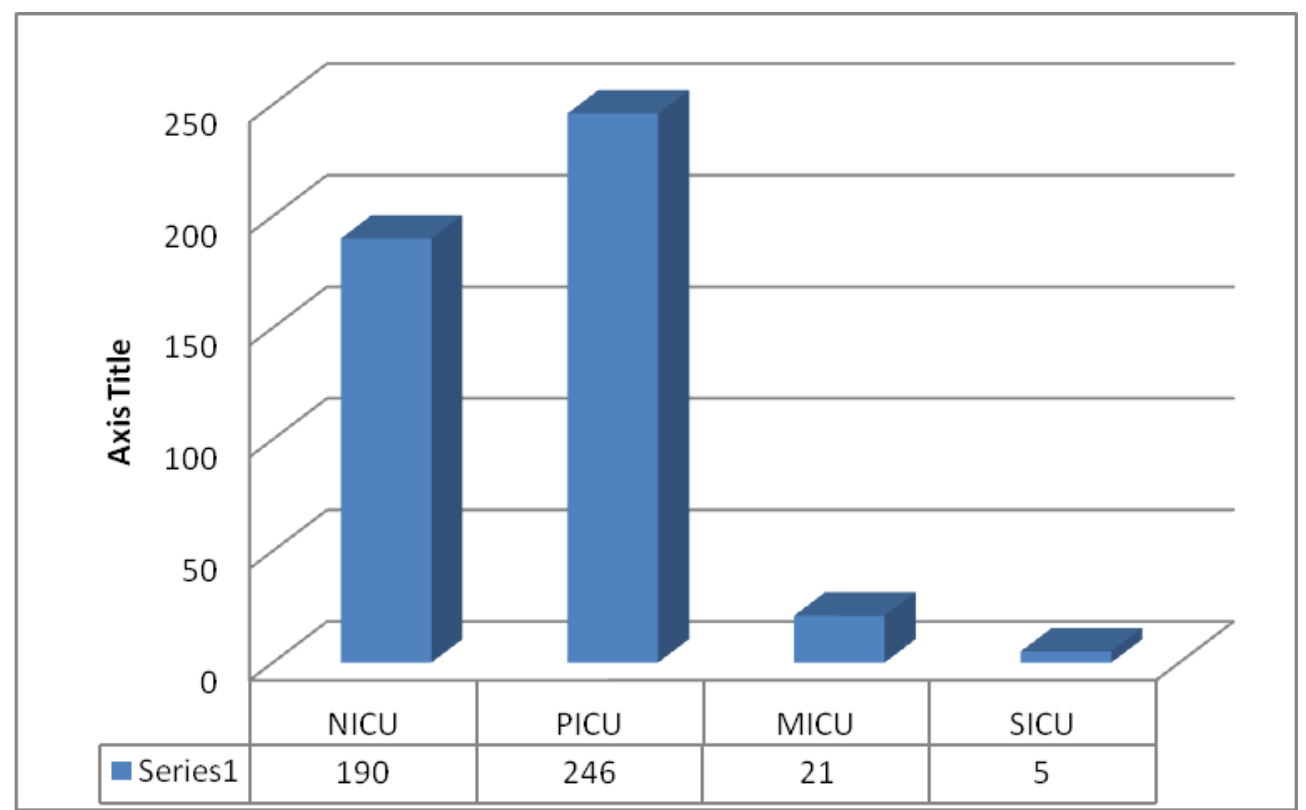


Fig.2 Comparison of isolates obtained in Neonates/ Children Vs Adults

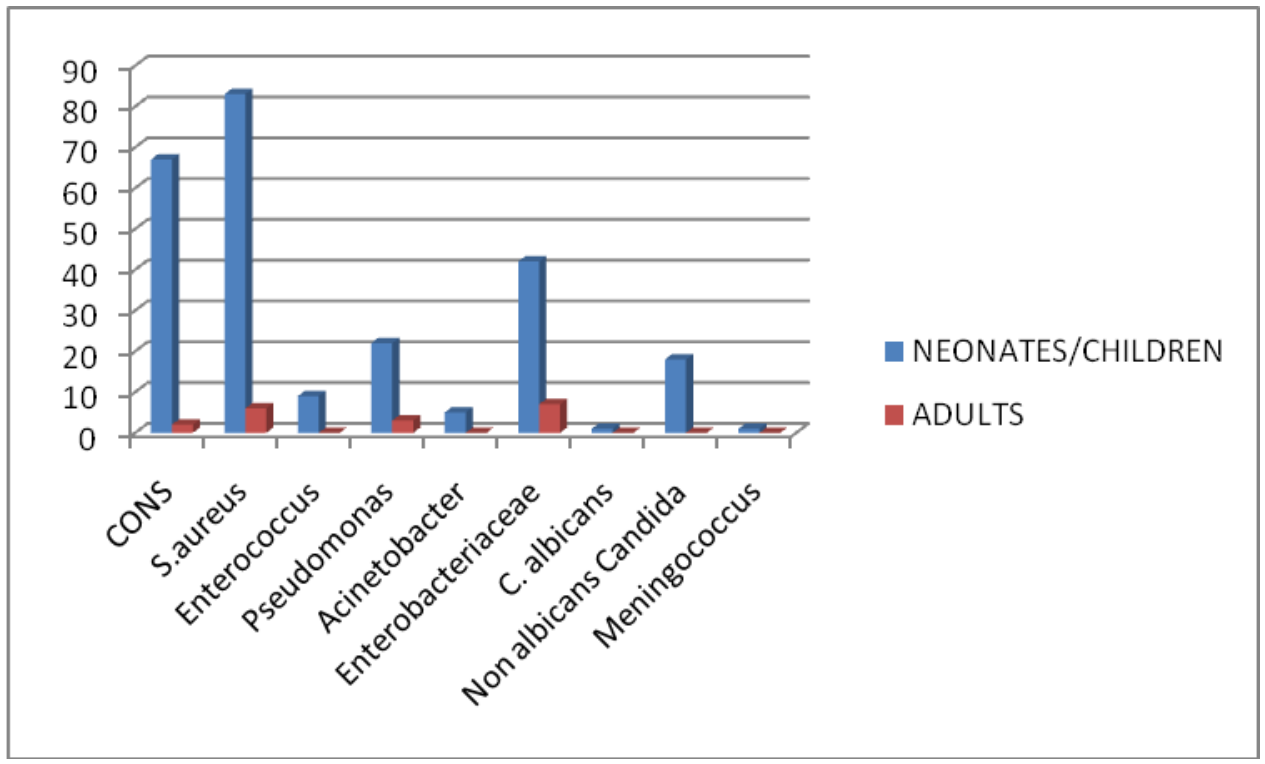

Fig.3 Distribution of various isolates.

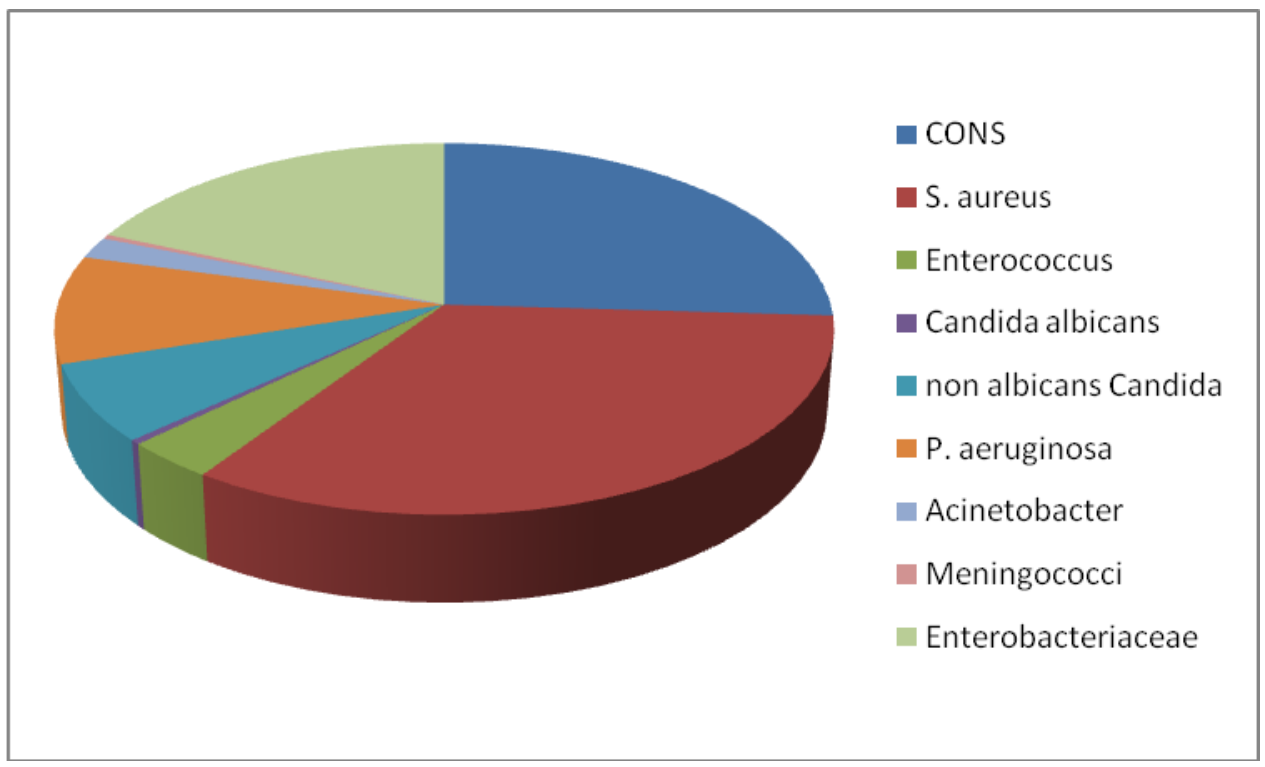

The common isolates in blood culture in our study were S. aureus $(33.5 \%)$ followed by GNBs (29.7\%), coagulase negative staphylococci (25.9\%), NAC (7.2\%), E.fecalis $(3.6 \%)$ and C. albicans $(0.4 \%)$.The findings are consistent with those of the previous studies conducted by (Karlowsky JA et al., 2004) and (Ben, et al., 2004). The results of our study demonstrate that blood culture Positivity rate in clinically suspected septicaemia cases was $57.6 \%$. Overall, $63.1 \%$ of septicaemia was caused by Grampositive and $29.7 \%$ by Gram negative bacilli and remaining $7.1 \%$ by Candida spp. this was in accordance with (Dagnew, Mulat et al., 2013) (M S Alam et al., 2011) and (Mamishi S et al., 2005) 
Like many other studies Coagulase-negative Staphylococcus were among the major blood culture isolates; however, given that CONS isolated from blood are often contaminants(> $85 \%$ are clinically insignificant) their antibiotic susceptibility was not determined. (Roy, et al., 2002)

In conclusion, Knowledge of the causative agents of bacteremia is useful in the selection of antimicrobials for empiric therapy. As $S$. aureus was the most common organism isolated, it suggests that good hygine practices by the patient,their attendants and the health care providers is very crucial especially in neonatal and paediatric ICUs. Also fairly high rate of isolating Pseudomona spp needs attention. The susceptibility data suggests that multidrug resistance is not a severe problem in local area yet as we are a new set up. But rational use of antibiotics should be practiced to avoid emergence of multi drug resistant. Reserved drugs need to be kept in reserved inventory to prevent their misuse and emergence of resistance. The in vivo susceptibility of the antimicrobials agents correlates well with the in vitro susceptibility reports and in our majority of the paediatric patients as a good therapeutic response was achieved. A changing trend in antibiotic sensitivity profile of the isolates need to be monitored as there is limited availability of newer drugs and the emergence of resistant bacteria far exceeds the rate of new drug development.

\section{References}

Bates, D.W., Goldman, L., Lee, T.H. 1991. Contaminant blood cultures and resource utilization. The true consequences of false-positive results. JAMA, 265: 365-9.

Ben, J.Z., Mahjoubi, F., Ben, H.Y. et al. 2004. Antimicrobial susceptibility and frequency of occurrence of clinical blood isolates in Tunisia (1993 1998). Pathol. Biol., 52: 82-8.

Bergmans, D.C., Bonten, M.J., Gaillard, C.A., et al. 1997. Indications for antibiotic use in ICU patients: a oneyear prospective surveillance. $J$. Antimicrob. Chemother., 39: 527535.

Brun-Buisson, C., Doyon, F., Carlet, J., Dellamonica, P., Gouin, F., Lepoutre, A., et al. 1995. Incidence, risk factors, and outcome of severe sepsis and septic shock in adults. A multicenter prospective study in intensive care units. French ICU Group for Severe Sepsis. JAMA, 274: 968-74.

Dagnew Mulat. et al. 2013. Bacterial Profile and Antimicrobial Susceptibility Pattern in Septicemia Suspected Patients Attending Gondar University Hospital, Northwest Ethiopia." BMC Res. Notes, 6: 283.

Eggimann, P., Pittet, D. 2001. Infection control in the ICU. Chest, 120: 205993.

Grace, C.J., Lieberman, J., Pierce, K., Littenberg, B. 2001. Usefulness of blood culture for hospitalized patients who are receiving antibiotic therapy. Clin Infect. Dis., 32: 1651-5.

Gaynes, R.P., Edwards, J.R., Jarvis, W.R., et al. 1996. Nosocomial infections among neonates in high risk nurseries in the United States. Pediatrics, 98: 357-361.

Jarvis, W.R., Edwards, J.R., Culver, D.H., Hughes, J.M., Horan, T., Emori, T.G. et al. 1991. Nosocomial infection rates in adult and pediatric intensive care units in the United States. National Nosocomial Infections Surveillance System. Am. J. Med., 91 (Suppl 3B): 185S-91S.

Karlowsky, J.A., Jones, M.E., Draghi, D.C. et al. 2004. Prevalence and antimicrobial susceptibilities of 
bacteria isolated from blood culture of hospitalised patients in the United States in 2002. Annals of Clin. Microbiol. Antimicrobials, 3: 7.

Mamishi, S., Pourakbari, B., Ashtiani, M.H., Hashemi, F.B. 2005. Frequency of isolation and antimicrobial susceptibility of bacteria isolated from bloodstream infections at Children's Medical Center, Tehran, Iran, 19962000. Int. J. Antimicrob. Agents, 26(5): 373-9.

Alam, M.S., P.K. Pillai, Prem Kapur and K.K. Pillai. 2011. Resistant patterns of bacteria isolated from bloodstream infections at a university hospital in Delhi. J. Pharm. Bioallied Sci., 3(4): 525-530.

NCCLS. 2002. Performance standards for antimicrobial susceptibility testing: Twelfth informational supplement M100-S12. NCCLS, Wayne, PA, USA.

Pittet, D., Tarara, D., Wenzel, R.P. 1994. Nosocomial bloodstream infection in critically ill patients: excess length of stay, extra costs, and attributable mortality. JAMA, 271: 1598-601.

Roy, I., Jain, A., Kumar, M., Agarwal, S.K. 2002. Bacteriology of neonatal septicaemia in a tertiary care hospital of northern India. Indian J. Med. Microbiol., 20: 156-9.

Vincent, J.L., Bihari, D.J., Suter, P.M., Bruining, H.A., White, J., NicolasChanoin, M.H. et al. 1995. The prevalence of nosocomial infection in intensive care units in Europe. Results of the European Prevalence of Infection in Intensive Care (EPIC) study. JAMA, 274: 639-44. 3.

Vincent, J.L., Sakr, Y., Sprung, C.L., Ranieri, V.M., Reinhart, K., Gerlach, H. et al. 2006. Sepsis in European intensive care units: results of the SOAP study. Crit. Care Med., 34: 344-53.

Weber, D.J., Raasch, R., Rutala, W.A. 1999. Nosocomial infections in the ICU. The growing importance of antibioticresistant pathogens. Chest, 115: 34S$41 \mathrm{~S}$.

\section{How to cite this article:}

Jyoti Sangwan, Pratibha Mane, Prakriti Vohra , Sumit Lathwal and A.K. Malik.2016. Bacteriologic Profile and Antimicrobial Resistance of Blood Culture Isolates of Saptecemic Patients from Various Intensive Care Units in a Teaching Tertiary Care Institute of Haryana. Int.J.Curr.Microbiol.App.Sci. 5(7): 599-608. doi: http://dx.doi.org/10.20546/ijcmas.2016.507.067 